\title{
Condutas de enfermagem à criança no pós-operatório de cirurgia cardíaca: análise das pesquisas
}

\author{
Postoperative nursing conduct towards children experiencing cardiac surgery: study analysis
}

Conductas de enfermería al niño en el postoperatorio de cirugía cardíaca: análisis de las investigaciones

Flávia Paula Magalhães Monteiro', Renata Pereira de Melo², Geane Felix de Souza ${ }^{3}$, Thelma Leite de Araujo ${ }^{4}$, Franscisca Elisângela Teixeira Lima ${ }^{5}$, Marcos Venícios de Oliveira Lopes ${ }^{6}$

\section{RESUMO}

As cardiopatias congênitas representam anomalias que incluem defeitos estruturais e funcionais no coração, os quais necessitam precocemente de intervenção de enfermagem à beira do leito. Objetivou-se analisar pesquisas que abordassem as condutas de enfermagem à criança no pós-operatório de cirurgia cardíaca. O presente trabalho trata-se de revisão integrativa da literatura em diferentes bases de dados, utilizando os descritores: Cuidados de enfermagem, Cirurgia cardíaca e Enfermagem pediátrica. Dos onze artigos identificados e analisados na íntegra, seis corresponderam aos critérios adotados no estudo, com evidências entre os níveis II e VI. As publicações culminaram nas condutas de prevenção, avaliação e recuperação ou reabilitação. Conclui-se que este estudo contribui para assistir no desenvolvimento de planos de cuidados de enfermagem no intuito de proporcionar melhorias na prática assistencial, com base em ações de promoção à saúde, com ênfase sobre avaliação da dor, verificação da temperatura invasiva e medidas não farmacológicas para manejo da dor, entre outras.

Descritores: Cuidados de Enfermagem; Cirurgia Cardíaca; Enfermagem Pediátrica; Revisão por Pares.

\section{ABSTRACT}

Congenital cardiopathies represent anomalies including structural and functional heart defects, which demand early nursing intervention at the bedside. The present study aimed at analyzing studies that addressed the nursing care provided to children postoperatively following cardiac surgery. It consists of an integrative review of the literature from different databases, using the descriptors: Nursing care; Cardiac surgery; and Pediatric nursing. From the eleven articles that were identified and completely analyzed, six corresponded to the criteria adopted in the study, with evidence levels ranging from level II to level VI. The publications highlighted recommendations concerning prevention, assessment, recovery and/or rehabilitation. It is concluded that this study contributes to the development of nursing care plans to provide improvements in healthcare practices, based on actions of health promotion, with emphasis on pain assessment, verification of the internal temperature and non-pharmacological measures to cope with pain, among others.

Descriptors: Nursing Care; Thoracic Surgery; Pediatric Nursing; Peer Review.

\section{RESUMEN}

Las cardiopatías congénitas representan anomalías que incluyen defectos cardíacos estructurales y funcionales, los cuales necesitan de pronta intervención de enfermería al pie del lecho. Se objetivó analizar investigaciones abordando las conductas de enfermería hacia niños en postoperatorio de cirugía cardíaca. Revisión integrativa de literatura según diferentes bases de datos, utilizando los descriptores: Cuidados de Enfermería, Cirugía Cardíaca y Enfermería Pediátrica. De once artículos identificados y analizados integralmente, seis correspondieron a los criterios adoptados en el estudio, con evidencias entre los niveles II y IV. Las publicaciones enfatizaron las conductas preventivas, de evaluación y recuperación o rehabilitación. Se concluye en que este estudio contribuye ayudando al desarrollo de planes de cuidados de enfermería con el objetivo de proporcionar mejorías en la práctica asistencial, con base en acciones de promoción sanitaria, con énfasis sobre la evaluación del dolor, verificación de temperatura invasiva y medidas no farmacológicas para manejo del dolor, entre otras.

Descriptores: Atención de Enfermería; Cirugía Torácica; Enfermería Pediátrica; Revisión por Expertos.

\footnotetext{
${ }^{1}$ Enfermeira, Mestre em Enfermagem. Discente do Programa de Pós-Graduação em Enfermagem da Universidade Federal do Ceará (UFC), nível Doutorado. Fortaleza, CE, Brasil. E-mail: flaviapmm@yahoo.com.br.

${ }^{2}$ Enfermeira, Doutora em Enfermagem. Enfermeira do Hospital da Mulher de Fortaleza. Fortaleza, CE, Brasil. E-mail: renatameloufc@yahoo.com.br.

${ }^{3}$ Farmacêutica, Mestre em Patologia. Discente do Programa de Pós-Graduação em Ciências Médicas da UFC, nível Doutorado. Fortaleza, CE, Brasil. Email: geanefelix@ig.com.br.

${ }^{4}$ Enfermeira, Doutora em Enfermagem. Professora Titular da UFC. Fortaleza, CE, Brasil. E-mail: thelmaaraujo2003@yahoo.com.br.

${ }^{5}$ Enfermeira, Doutora em Enfermagem. Professora Adjunto da UFC. Fortaleza, CE, Brasil. E-mail: felisangela@yahoo.com.br.

${ }^{6}$ Enfermeiro, Doutor em Enfermagem. Professor Adjunto da UFC. Fortaleza, CE, Brasil. E-mail: marcos@ufc.br.
} 


\section{INTRODUÇÃO}

As cardiopatias congênitas representam anomalias, cuja expressão clínica inclui defeitos estruturais e funcionais no coração que atingem aproximadamente oito em cada 1.000 nascidos vivos na população americana. Diante deste fato, avalia-se que cerca de 650.000 a 1,3 milhão de americanos na idade adulta convivem atualmente com defeitos cardiovasculares e pelo menos 35 defeitos já foram identificados ${ }^{(1)}$. No Brasil, estima-se o surgimento de 28.846 novos casos de cardiopatia congênita por ano(2).

Comumente, nos diferentes serviços de saúde com especialidade cardiológica pediátrica, tem-se observado aumento progressivo de crianças em idade precoce com sinais e sintomas de cianose, taquipneia, sopro cardíaco e arritmias, os quais exigem cuidados cirúrgicos ou ambulatoriais $^{(3)}$. Ao serem diagnosticadas, as crianças portadoras de cardiopatias congênitas são acompanhadas e avaliadas quanto à necessidade de intervenção cirúrgica corretiva ou paliativa e à presença de alterações hemodinâmicas. De modo geral, o tratamento é fundamental para prevenir o desenvolvimento de doenças associadas e proporcionar melhor qualidade de vida aos afetados.

Nesse contexto, os defeitos provocados pelas cardiopatias são causas de morte entre as crianças no primeiro ano de vida, especialmente no período neonatal em virtude da gravidade da doença e das modificações fisiológicas muito comuns nessa fase. Em face disso, as indicações e a idade da criança submetida à cirurgia tem se modificado atualmente.

Como observado, o momento mais adequado para a correção dessas cardiopatias depende do tipo e da natureza do defeito congênito, da presença e da severidade dos sintomas, visto que o tratamento cirúrgico assegura melhores chances de sobrevida, torna a função cardíaca mais próxima do normal e cria condições para a reversão de alterações secundárias ${ }^{(4)}$.

O período pós-operatório das crianças portadoras de cardiopatias congênitas envolve condutas de enfermagem realizadas à beira do leito numa unidade de cuidados críticos. Tais condutas incluem monitorização de sinais vitais, avaliação hemodinâmica e laboratorial, suporte e ajustes ventilatórios, suporte nutricional, manutenção de cateteres para infusão de fármacos vasoativos e hemocomponentes, avaliação da incisão cirúrgica quanto à presença de sangramentos, acompanhamento do débito urinário, avaliação das perdas pelo controle hidroeletrolítico, inclusive procedimentos específicos relacionados à hemodiálise ou diálise peritoneal(5-7). Somando-se a isso, o enfermeiro exerce importante papel no apoio aos pais, mediante fornecimento de informações quanto aos procedimentos executados, desenvolvimento da cirurgia e evolução da criança na unidade pós-operatória infantil.

Nesse momento, o enfermeiro tem o compromisso de intervir de forma criteriosa sobre as principais necessidades da criança, bem como aliar suas experiências clínicas às evidências contidas na literatura para fortalecer as devidas condutas à tomada de decisão.

Diante da necessidade de literatura que aponte a melhor evidência disponível, esse estudo teve como objetivo analisar pesquisas que abordem as condutas de enfermagem à criança no período pós-operatório de cirurgia cardíaca, na tentativa de ampliar o conhecimento e contribuir para melhorias na assistência pós-operatória.

\section{METODOLOGIA}

Para o desenvolvimento deste estudo, procedeu-se à revisão integrativa da literatura de acordo com as seguintes etapas: formulação do problema, busca na literatura, avaliação e análise dos dados e apresentação(8).

A etapa de formulação do problema incluiu a identificação do interesse, o propósito da revisão integrativa e a elaboração da pergunta norteadora. Assim, o problema de interesse foi semelhante a pergunta norteadora elaborada para direcionar a busca: quais as condutas de enfermagem realizadas no pósoperatório de cirurgia cardíaca em crianças?

Como critérios de inclusão estabeleceram-se: artigos de pesquisa que apresentassem texto completo, disponíveis eletronicamente nas bases de dados ou nos periódicos do portal CAPES, nos idiomas inglês, espanhol ou português, publicados até dezembro de 2011 (período da coleta dos dados). Além disso, a busca restringiu-se às produções que envolvessem crianças e retratassem o período pós-operatório de cirurgia cardíaca. Quanto aos critérios de exclusão, definiram-se as publicações do tipo editorial, de revisão narrativa ou integrativa, resumo em anais de evento, dissertação e tese, bem como publicações repetidas. 
A seleção foi feita de forma ordenada nas bases de dados Scopus, National Library of Medicine (PubMed), Cumulative Index to Nursing and Allied Health Literature (CINAHL with full text), Centro Latino-Americano e do Caribe de Informações em Ciências da Saúde (LILACS) e Cochrane, considerando-se suas especificidades.

A busca nas bases de dados foi realizada mediante a identificação dos descritores no Medical Subject Headings (MeSH) e nos Descritores em Ciências da Saúde (DeCS), considerando-se os termos presentes na pergunta norteadora. Assim, identificaram-se os termos Nursing
Care, Cardiac Surgery e Pediatric Nursing e Cuidados de Enfermagem/Atención de Enfermería/Nursing Care; Cirurgia Cardiaca (sinônimo)/Cirugia e Enfermagem Pediátrica/ Enfermería Pediátrica/ Pediatric Nursing, respectivamente. Ressalta-se que para todas as bases utilizou-se o cruzamento dos três descritores, exceto para a pesquisa na base de dados LILACS, na qual o cruzamento dos três termos não gerou produções. Dessa forma, optou-se pelo cruzamento dos descritores Cuidados de Enfermagem e Cirurgia Cardíaca, conforme se observa na Figura 1.

Busca de dados

\begin{tabular}{|c|}
\hline \multicolumn{1}{|c|}{ SCOPUS } \\
Nursing Care \\
Cardiac Surgery \\
Pediatric Nursing \\
\hline \multicolumn{1}{|c|}{ 46 produções: } \\
13 de enfermagem \\
\hline 3 sem resumo \\
$\mathbf{5}$ fora do tema \\
4 revisões \\
$\mathbf{1}$ selecionada \\
\\
\end{tabular}

\begin{tabular}{|c|}
\hline $\begin{array}{c}\text { PUBMED } \\
\text { Nursing Care, } \\
\text { Cardiac Surgery } \\
\text { Pediatric Nursing }\end{array}$ \\
\hline 71 produções \\
\hline $\begin{array}{l}40 \text { sem resumo } \\
15 \text { fora do tema } \\
7 \text { indisponíveis } \\
3 \text { repetidas } \\
5 \text { revisões } \\
1 \text { selecionada }\end{array}$ \\
\hline
\end{tabular}

\begin{tabular}{|c|}
\hline $\begin{array}{c}\text { CINAHL } \\
\text { Nursing Care, } \\
\text { Cardiac Surgery } \\
\text { Pediatric Nursing }\end{array}$ \\
\hline 74 produções \\
\hline 40 fora do tema \\
6 sem resumo \\
4 editoriais \\
2 repetidas \\
3 indisponíveis \\
3 anais \\
1 resumo de livro \\
8 revisões \\
$\mathbf{7}$ selecionadas \\
\hline
\end{tabular}

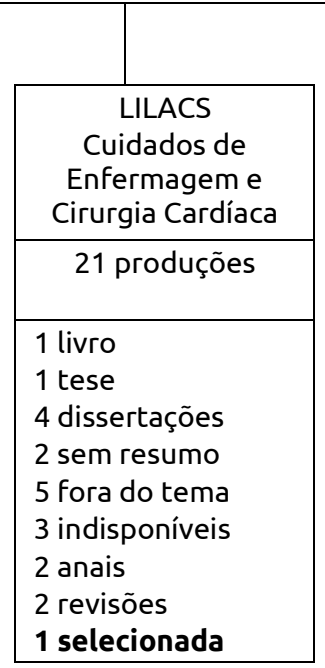

\begin{tabular}{|c|}
\hline $\begin{array}{c}\text { COCHRANE } \\
\text { Nursing Care, Cardiac } \\
\text { Surgery } \\
\text { Pediatric Nursing }\end{array}$ \\
\hline 67 produções \\
\hline 1 repetida \\
65 fora do tema \\
1 selecionada \\
\\
\end{tabular}

Figura 1: Percurso metodológico para seleção das produções analisadas.

Todos os artigos identificados foram submetidos às duas etapas de avaliação. A primeira dirigia-se à apreciação do título e do resumo, com vistas a identificar sua adequação à pergunta norteadora e aos critérios de inclusão e exclusão pré-estabelecidos. A segunda etapa apontou a extração das características metodológicas relevantes, por meio de instrumento adaptado, o qual contemplou os seguintes aspectos: tipo de estudo; autores; intervenção aplicada; resultados; recomendações/conclusões ${ }^{(9)}$.

Em seguida, analisaram-se os artigos quanto ao ano de publicação, tipo de estudo, objeto de estudo, níveis de evidência ${ }^{(10)}$ e condutas de enfermagem.

\section{RESULTADOS E DISCUSSÃO}

Dos 11 artigos analisados na íntegra, seis corresponderam aos critérios adotados. Ressalta-se que apesar da busca ter compreendido estudos publicados até o mês de dezembro de 2011 (período da coleta dos dados), a amostra abrangeu apenas os artigos publicados de 1999 a 2009. Com relação ao tipo de estudo, dois trabalhos eram observacionais e descritivos; um exploratório e descritivo; e três experimentais (ensaios clínicos), dos quais dois randomizados. No tocante aos níveis de evidências, dois trabalhos foram classificados no nível II, um no nível III e três no nível VI.

A seguir, o Quadro 1 apresenta a caracterização das produções quanto ao objeto de estudo e às condutas de enfermagem. Aqueles com ampla abrangência de condutas foram incluídos em mais de uma categoria. 
Quadro 1: Caracterização dos artigos quanto ao objeto de estudo e condutas de enfermagem.

\begin{tabular}{|c|c|c|c|c|}
\hline Referência & $\begin{array}{l}\text { Nível de } \\
\text { evidência }\end{array}$ & Delineamento do estudo & Objeto de estudo & Condutas de enfermagem (Resultados dos estudos) \\
\hline $\begin{array}{l}\text { 1. Puntillo KA, Jacob E. (1999). A survey of nursing } \\
\text { practice in the assessment and management of } \\
\text { pain in children. Pediatric nursing, } 25(3): 278-86 \text {. }\end{array}$ & $\mathrm{VI}$ & $\begin{array}{l}\text { Observacional } \\
\text { descritivo/Abordagem } \\
\text { quantitativa }\end{array}$ & $\begin{array}{l}\text { Percepção das enfermeiras } \\
\text { na avaliação e manejo da dor } \\
\text { em crianças. }\end{array}$ & $\begin{array}{l}\text { 1) Avaliar a dor incluindo expressão verbal, facial e } \\
\text { linguagem corporal; bem como as seguintes mudanças: } \\
\text { fisiológicas, pele, estado neurológico, afeto, humor, } \\
\text { atividades de vida diária; condição do paciente e entrada } \\
\text { dos pais no setor; 2) Administrar medicamentos para o } \\
\text { alívio da dor; 3) Adotar medidas não-farmacológicas para } \\
\text { o manejo da dor. }\end{array}$ \\
\hline $\begin{array}{l}\text { 2. Maxton FJC, Justin L, Gillies D. (2004). } \\
\text { Estimating core temperature in infants and } \\
\text { children after cardiac surgery: a comparison of six } \\
\text { methods. Issues and innovations in nursing } \\
\text { practice, } 45(2): 214-222 \text {. }\end{array}$ & III & $\begin{array}{l}\text { Ensaio clínico controlado sem } \\
\text { randomização }\end{array}$ & $\begin{array}{l}\text { Monitorização } \\
\text { temperatura central da } \\
\text { crianças criticamente } \\
\text { doentes por meio de } \\
\text { distintos métodos e locais. }\end{array}$ & $\begin{array}{l}\text { 1) Monitorizar a temperatura: nasofaríngea, axilar, retal, } \\
\text { timpânica, bexiga sanguínea (artéria pulmonar). }\end{array}$ \\
\hline $\begin{array}{l}\text { 3. Harbaugh BL, Tomlinson PS, Kirschbaum M. } \\
\text { (2004). Parents' perceptions of nurses' caregiving } \\
\text { behaviors in the pediatric intensive care unit. } \\
\text { Issues in Comprehensive Pediatric Nursing, } \\
27: 163-178 \text {. }\end{array}$ & VI & $\begin{array}{l}\text { Descritivo/Abordagem } \\
\text { qualitativa }\end{array}$ & $\begin{array}{lr}\text { Percepção } & \text { do } \\
\text { comportamento } & \text { dos } \\
\text { enfermeiros pelos pais. } & \end{array}$ & $\begin{array}{l}\text { 1)Informar aos pais e crianças acerca das tecnologias do } \\
\text { ambiente e procedimentos; 2) Orientar os pais sobre as } \\
\text { condições de saúde da criança; 2)Monitorizar a criança; } \\
\text { 3)Garantir a presença dos pais na UTI; 4) Favorecer a } \\
\text { comunicação entre pais e enfermeiros }\end{array}$ \\
\hline $\begin{array}{l}\text { 4. Arino M, Barrington JP, Morrison } A L \text {, Gillies } D \text {. } \\
\text { (2004). Management of the changeover of } \\
\text { inotrope infusions in children. Intensive and } \\
\text { Critical Care Nursing, } 20: 275-280 \text {. }\end{array}$ & II & $\begin{array}{l}\text { Ensaio clínico controlado } \\
\text { randomizado }\end{array}$ & $\begin{array}{l}\text { Método de infusão } \\
\text { inotrópica: mudança rápida } \\
\text { ou dupla infusão. }\end{array}$ & 1) Administrar drogas vasoativas \\
\hline $\begin{array}{l}\text { 5.Chu Y-Chu, Lin Su-Man, Hsieh Ying-Chou, Chan } \\
\text { Kwork-Hon, Tsou Mei-Yung. (2006). Intraoperative } \\
\text { administration of tramadol for postoperative } \\
\text { nurse-controlled analgesia resulted in earlier } \\
\text { awakening and less sedation than morphine in } \\
\text { children after cardiac surgery. Anesth Analg, 102: } \\
1668-73 \text {. }\end{array}$ & II & $\begin{array}{l}\text { Ensaio clínico controlado } \\
\text { randomizado }\end{array}$ & $\begin{array}{l}\text { Avaliação da resposta da } \\
\text { criança no pós-operatório } \\
\text { quanto à analgesia } \\
\text { controlada pela enfermeira } \\
\text { no intraoperatório. }\end{array}$ & $\begin{array}{l}\text { 1) Monitorizar a criança nos aspectos hemodinâmicos e } \\
\text { respiratórios; 2) Administrar medicamentos; 3) avaliar a } \\
\text { dor e condições da criança para iniciar a alimentação. }\end{array}$ \\
\hline $\begin{array}{l}\text { 6. Hatler C, Buckwald L, Salas-Allison Z, Murphy- } \\
\text { Taylor C. (2009). Evaluating central venous } \\
\text { catheter care in a pediatric intensive care unit. } \\
\text { American Journal of Critical Care, } 8(6): 514-521 \text {. }\end{array}$ & VI & $\begin{array}{l}\text { Exploratório } \\
\text { observacional/Abordagem } \\
\text { quantitativa }\end{array}$ & $\begin{array}{llr}\text { Cobertura utilizada na } \\
\text { inserção de cateteres } \\
\text { venosos centrais. }\end{array}$ & $\begin{array}{l}\text { 1) Avaliar o local de inserção do cateter venoso central; } \\
\text { 2) trocar cobertura do catéter; 3)Lavar as mãos; 4)Avaliar } \\
\text { a perfusão. }\end{array}$ \\
\hline
\end{tabular}


Adiante, os resultados serão discutidos com base nas ações de enfermagem que representaram Condutas de Prevenção, Condutas de Avaliação e Condutas de Recuperação ou Reabilitação.

\section{Condutas de prevenção}

Representaram ações de enfermagem que antecediam a ocorrência de possíveis alterações na criança ou forneciam um canal de comunicação entre enfermeiro, pais/responsáveis e crianças.

Ditas condutas de enfermagem foram aplicadas após procedimento cirúrgico, desde o momento da admissão da criança na unidade de recuperação ou terapia intensiva até sua alta. Compreenderam: avaliar os locais de inserção de cateter venosos; administrar drogas vasoativas; avaliar a perfusão venosa; monitorar a temperatura e os aspectos hemodinâmicos e respiratórios.

Nesse ensejo, a admissão da criança, na unidade de terapia intensiva após o procedimento cirúrgico cardíaco, é um momento de grande expectativa e tensões que requer o monitoramento do paciente nas primeiras 24 horas. Inicialmente, a criança é posicionada no leito e verificam-se o local de inserção dos acessos, cateter para pressão arterial direta, bem como monitor cardíaco e ventilador mecânico, os quais devem ser calibrados, porquanto a manutenção desses parâmetros torna-se vital para a tomada de decisões do enfermeiro $^{(11)}$.

Além disso, durante a admissão, a interação entre os profissionais da equipe de saúde é essencial, pois todos os dados referentes à criança no pré e transoperatório devem ser compartilhados pelos profissionais responsáveis por seu cuidado no pós-operatório(12).

Adicionalmente, o enfermeiro supervisiona os acessos centrais e periféricos quanto à acessibilidade e retorno. Em seguida, durante a administração de drogas vasoativas, deve observar o tempo adequado para troca dos dispositivos flexíveis que permitem a infusão simultânea de soluções compatíveis em uma mesma via de acesso venoso, e a necessidade de renovação dessas drogas preservando sua estabilidade, para prevenir possíveis obstruções no sistema e instabilidades hemodinâmicas na criança.

Acerca da renovação dos fármacos ou troca de bombas infusoras, estudo experimentou método que resultou na menor alteração da frequência cardíaca e pressão arterial. Os autores concluíram não existir diferença clinicamente significante entre os métodos de troca rápida e o de dupla infusão, mas recomendaram o de mudança rápida por ser mais ágil, simples e acarretar menores riscos de tolerância ${ }^{(13)}$.

Concluída a avaliação dos acessos e das drogas, o enfermeiro verifica o débito urinário e a quantidade de sangue eliminada pelos drenos do mediastino e torácico, e ao mesmo tempo faz ausculta pulmonar com vistas a avaliar presença de ruídos adventícios, expansibilidade torácica e necessidade de aspiração para evitar a estase das secreções e obstruções das vias aéreas ${ }^{(14)}$.

Após estabilização da criança, as condutas de enfermagem relacionadas à promoção da saúde familiar são fornecidas, especificamente sobre a preparação dos familiares acerca da resposta da criança à cirurgia e suas condições na unidade. Nesse sentido, foram identificadas as condutas: garantir a presença dos pais no setor; informar aos pais e às crianças acerca das tecnologias do ambiente e de procedimentos; e favorecer a comunicação entre pais e enfermeiros.

O cuidado de enfermagem em cirurgia cardíaca é complexo, sobretudo por ter o intuito de melhorar a sobrevida e a qualidade de vida da criança. Dessa forma, o cuidado deve compreender todas as ações do enfermeiro direcionadas ao cliente desde o momento do conhecimento da cirurgia, quando se iniciam as orientações pré-operatórias ao cliente e seus familiares ${ }^{(12)}$.

Um dos estudos analisados, de abordagem qualitativa, realizado com dez crianças de sete a dez anos de idade, submetidas à cirurgia pela primeira vez, identificou a necessidade de desempenho do cuidado adequado a sua faixa etária, respeitando as diferentes fases do desenvolvimento da criança(15).

Outro estudo, realizado nos Estados Unidos com pais ( $n=9)$ e mães $(n=10)$ de dez crianças e evidenciou a avaliação do comportamento de enfermeiras na unidade de terapia intensiva pelos pais das crianças hospitalizadas e revelou diferentes respostas demonstradas por estas profissionais sobre a garantia da qualidade do cuidado, atenção, afeto, toques e gestos de compreensão da criança com fácies de dor. Segundo os pais, as enfermeiras mostravam-se solícitas e permitiam plena comunicação sobre a condição da criança e procedimentos executados. Assim, os pais se sentiam mais confortáveis e menos ansiosos ${ }^{(16)}$. 
Corroborando, estudo aponta a utilização de estratégias de ensino, como álbum seriado, filmes educativos e terapias ocupacionais para minimizar os sentimentos de medo e ansiedade e proporcionar resultados satisfatórios durante o tratamento, tendo em vista que quando as mães/responsáveis estão em um ambiente desconhecido, é necessário que a equipe de enfermagem forneça as informações básicas, assim como oriente e questione sobre possíveis dúvidas. $\mathrm{O}$ que parece claro para a equipe, nem sempre é claro para as mães ${ }^{(17)}$.

Assim, por meio de plano de ensino contendo desenho de boneco ou figuras, o enfermeiro pode demonstrar os possíveis locais de acessos, sondas, tubo orotraqueal, incisão cirúrgica, drenos, e explicar a necessidade de cada procedimento ${ }^{(14)}$. Neste momento, os pais são encorajados a fazer perguntas e a explorar qualquer instrumento adicional no setor. Tais medidas são profiláticas e tentam aproximar a família ao ambiente hospitalar e torná-la coparticipante desse momento de cuidados.

Além disso, a ação do enfermeiro centrada na família inclui a preparação para alta, mediante orientações nutricionais, administração de medicamentos e o ensino para identificação de sinais e sintomas de descompensação cardíaca e alterações respiratórias ${ }^{(18)}$.

\section{Condutas de avaliação}

Incluíram a avaliação da dor, mediante a expressão verbal, facial e linguagem corporal, mudanças nas condições fisiológicas da criança, na pele, estado neurológico, afeto, humor, atividades de vida diária; da entrada dos pais no setor; das condições da criança para iniciar a alimentação; do local de inserção do cateter venoso central e da perfusão venosa.

Sobre a avaliação da dor, estudo mencionou atenção especial dos enfermeiros à dor referida ou demonstrada pelas crianças. Consoante a literatura revela, a avaliação e o manejo da dor em crianças têm sido amplamente empregado pelas enfermeiras que atuam nas unidades de terapias intensivas pediátricas ${ }^{(19)}$.

Em contrapartida, o conhecimento e os novos instrumentos de avaliação da dor não têm sido aplicados na prática porque as enfermeiras desconhecem a farmacodinâmica das drogas analgésicas; não reconhecem o valor da combinação entre as terapias farmacológicas e não-farmacológicas, bem como não utilizam rotineiramente escalas de avaliação da dor ${ }^{(19)}$. Além disso, a dor pode ser influenciada pelo ambiente, experiências prévias, cultura, atitude dos pais e comportamento de choro e sono, dificultando consideravelmente a avaliação da enfermeira(20).

A dor constitui o quinto sinal vital, especificamente quando se trata de crianças e portadores de doenças crônicas. Nesse sentido, a Joint Commission on Accreditation of Healthcare Organizations ${ }^{(21)}$, publicou norma que descreve sua avaliação por meio do questionamento da intensidade, início, local, irradiação, periodicidade, tipo de dor, duração e fatores desencadeantes.

No tocante à avaliação da alimentação, o enfermeiro deve manter-se atento ao início precoce da dieta, pois a criança portadora de cardiopatia congênita está notadamente em risco nutricional, determinado por problemas como dispneia, crises de hipóxia e dificuldade de sucção durante a alimentação oral(22). Na condição pós-operatória, muitas crianças requerem sondas nasogástrica ou orogástrica até se sentirem em condições para iniciar alimentação oral(16). Nesse cuidado, a enfermeira avalia a tolerância da criança, a presença, coloração e a quantidade do resíduo gástrico, e também a presença de ruídos hidroaéreos para começar a alimentação.

Acerca dos acessos periféricos e centrais com múltiplos lúmens, avalia-se a integridade e a perfusão. De acordo com a necessidade da infusão, um lúmen é reservado para infusão de drogas vasoativas; um segundo, para fluidos, tais como hidratação com eletrólitos, e um terceiro, para sedação contínua ${ }^{(11)}$.

Segundo se observa na prática assistencial, ao administrar fármacos endovenosos e outros fluidos, o enfermeiro avalia o local de inserção do cateter venoso quanto à presença de infiltrados, sinais característicos de inflamação, bem como infecção bacteriana, com vistas à prevenção de infecções no sítio do cateter. Estas ações devem ser supervisionadas e orientadas pela comissão de controle de infecção hospitalar da instituição.

Durante as primeiras 24 horas, comumente, a criança pode apresentar febre e alterações nas células brancas no hemograma, decorrentes da resposta inflamatória sistêmica pela cirurgia. Portanto, ainda não é estabelecida a infecção, pois, na suspeita desta, realizam-se hemoculturas e cultura do cateter ${ }^{(23)}$. 
Dessa forma, segundo estudos ora analisados, nas medidas profiláticas contra infecções, o enfermeiro deve avaliar diariamente o cateter ao administrar os fluidos e trocar os curativos; lavar as mãos por 15 segundos; reduzir a manipulação do cateter; usar luvas e máscaras; limpar a área com clorexidine e utilizar gazes embebidas em álcool etílico.

Ainda sobre a avaliação, identificaram-se condutas relacionadas à monitorização da temperatura, aspectos hemodinâmicos e respiratórios da criança. No tocante a isso, um estudo aponta que deve ser avaliada a presença de hipotermia, sobretudo, na primeira hora do pósoperatório(11).

No concernente ao controle da temperatura, as crianças submetidas à cirurgia demandam manutenção da normotermia, pois seu aumento exige maior consumo de oxigênio, ocasionando queda na saturação. Ademais, a taquicardia associada à febre e ao estresse aumenta o trabalho da bomba cardíaca em recuperação(18). Somado a isso, o estresse proveniente da cirurgia e a presença dos fatores como anestesia, hipotermia e incisão cirúrgica aumentam a incidência de arritmias no período pós-operatório e, por conseguinte, provocam alterações hemodinâmicas.

No entanto, ainda são frequentes as discussões sobre os locais de aferição da temperatura mais fidedignos. Rotineiramente, observa-se a medida em locais de fácil acesso e não-invasivos, selecionados conforme a condição de saúde da criança. Por outro lado, a medida da temperatura por cateter na artéria pulmonar é considerada mais acurada e referenciada como padrão-ouro.

\section{Condutas de recuperação ou reabilitação}

Especificaram-se ações interdependentes de outras condutas profissionais em que o enfermeiro preocupa-se com o restabelecimento das condições de saúde da criança. Dessa forma, a implementação dessas condutas está destinada à continuidade do tratamento, com ênfase sobre o processo curativo. São elas: administração de medicamentos para o alívio da dor; administração de drogas vasoativas e outros medicamentos; e adoção de medidas não-farmacológicas para o manejo da dor e troca de cobertura do cateter.

A criança portadora de cardiopatias menos complexas que não cursam com disfunção ventricular e/ou hipertensão pulmonar raramente desenvolve baixo débito no pós-operatório, ao contrário das mais complexas, geralmente acompanhadas de insuficiência cardíaca congestiva. Se a criança não apresenta nenhum distúrbio ácido- básico ou metabólico, está bem oxigenada, com volemia normal, dor controlada, sem alterações da temperatura, mas manifesta alteração da contratilidade miocárdica, está indicado o uso de drogas inotrópicas ${ }^{(23)}$.

Essas drogas são administradas isoladamente ou combinadas, conforme a dose e a resposta da criança a cada uma delas. Quando os agentes inotrópicos usados isoladamente não melhoram a contratilidade miocárdica, utilizam-se os vasodilatadores, os quais são administrados pelo enfermeiro, cuidadosamente atento aos parâmetros da frequência cardíaca e pressão arterial média, bem como às eventuais alterações da resposta da criança à dose prescrita e à necessidade de desmame.

As medidas não-farmacológicas são divulgadas entre os pesquisadores com a ressalva de grande necessidade de preparo do enfermeiro para o manejo e alívio da dor. Como exemplos, mencionam-se as técnicas de distração e relaxamento, incluindo o uso da musicoterapia, imagens guiadas pela enfermeira, medidas de conforto, tais como toque e o uso de cobertores (cueiros), como medidas de alívio da dor ${ }^{(19)}$.

Além dessas, outras estratégias são utilizadas: massagens relaxantes, bolsa de água morna, sucção nãonutritiva, aconchego do colo tanto do profissional como do pai/mãe e diminuição de estímulos auditivos e visuais. Nesse sentido, observa-se que o controle para o alívio do desconforto e sofrimento ainda é umas das grandes preocupações cotidianas do enfermeiro. Este implementa importante papel na avaliação da dor, orientação e implementação da terapêutica, bem como na eficácia do tratamento empregado.

Finalmente, a troca de coberturas é uma ação de enfermagem executada rotineiramente. Nesse contexto, um dos estudos analisados abordou a avaliação de dois tipos de coberturas transparentes para cateter venoso central em crianças que se diferenciavam pela presença de uma substância denominada Biopatch plus ${ }^{\circledR}$. Ao final, os autores concluíram que nenhum dos participantes desenvolveu infecção sanguínea relacionada ao cateter; não houve diferença significante $(p>0,05)$ entre o uso das coberturas e o número de dias com febre e o tempo de permanência do cateter no local; e a quantidade de medicações administradas em 24 h foi preditiva $(p<0,001)$ 
para desenvolvimento de febre, sugerindo que o número de manipulações do cateter pode predispor à infecção.

\section{CONSIDERAÇÕES FINAIS}

Seis artigos corresponderam aos critérios adotados no estudo e foram publicados no período de 1999 a 2009, destacando-se o ano de 2004, com três artigos. Devido ao método de revisão utilizado, não foi estabelecido limite temporal para seleção dos estudos. As publicações apresentaram delineamento observacional e descritivo; exploratório e descritivo; e experimental (ensaio clínico), dois deles com randomização. Entre os estudos, o nível VI de evidência e a natureza quantitativa predominaram.

As categorias ora elaboradas se referiam às condutas de prevenção; condutas de avaliação e condutas de recuperação ou reabilitação da saúde da criança, nas quais o enfermeiro executa atividades semelhantes com diferentes facetas.

\section{REFERÊNCIAS}

1. Heart Disease and Stroke Statistics - 2010 update: a report from the American Heart Association. Circulation. 2010;121:e46-e215.

2. Pinto Junior VC, Daher CV, Sallum FS, Jatene MB, Croti UA. Situação das cirurgias cardíacas congênitas no Brasil. Braz J Cardiovasc Surg. 2004;19(2):3-6.

3. Brooks PA, Penny DJ. Management of the sick neonate with suspected heart disease. Early Human Development. 2008;84:155-9. 4. Jansen D, Silva KVPT, Novello R, Guimarães TCF, Silva VG.

Assistência de enfermagem à criança portadora de cardiopatia. Rev SOCERJ. 2000;13(1):22-9.

5. Batista JFC, Silva ACSS, Azeredo NA, Moura SM, Mattos VZ. A enfermagem no cuidado integrado ao recém-nascido com cardiopatia congênita: relato de caso. Online Braz J Nurs. [Internet] 2005 [cited 2008 abr 16]. 4(1). Available from:

http://www.uff.br/objnursing/index.php/nursing/issue/archive 6. Ferreira FG, Gengo e Silva RC, Gonçalves CHB, Palomo JSH. Pósoperatório imediato de cirurgia cardíaca pediátrica: rotina de enfermagem para admissão do paciente na Unidade de Terapia Intensiva. Rev Bras Cir Cardiovasc. 2011;26(2):301-2.

7. Souza $P$, Scatolin BE, Ferreira DLM, Croti UA. A relação da equipe de enfermagem com a criança e a família em pós-operatório imediato de cardiopatias congênitas. Arq Ciênc Saúde. 2008; 15(4): 163-9.

8. Whittemore R, Knafl K. The integrative review: update methodology. J Adv Nurs. 2005; 52(5):546-553.

9. Ursi ES, Galvão CM. Prevenção de lesões de pele no perioperatório: revisão integrativa da literatura. Rev Latino-am Enfermagem. 2006; 14(1):124-31.

10. Melnyk BM, Fineout-Overholt E. Evidence-based practice in nursing \& healthcare: a guide to best practice. Philadelphia: Wolters Kluwer, Lippincott Williams \& Wilkins, 2011.

11. Simarro JVC. Cirugía cardíaca: cuidados iniciales de enfermería en el ingreso en la unidad de cuidados intensivos. Rev Cuba Enferm. 2002; 18(1):72-7.

12. Cavalcanti ACD, Coelho MJ. A linguagem como ferramenta do cuidado do enfermeiro em cirurgia cardíaca. Esc Anna Nery Rev Enferm. 2007; 11(2): 220-6.
Com base no exposto, esse estudo fornece subsídios para assistir no desenvolvimento de planos de cuidados de enfermagem no intuito de proporcionar melhorias no desenvolvimento da prática assistencial, com base em ações de promoção à saúde. Assim, na categoria Condutas de prevenção, destacaram-se: a recomendação do método de troca rápida da bomba infusora na tentativa de minimizar instabilidades hemodinâmicas; o envolvimento da tríade família-criança-enfermeiro e a utilização de estratégias de ensino com o intuito de incentivar a coparticipação da família no cuidado.

Quanto às Condutas de avaliação, identificaram-se a investigação da dor, como quinto sinal vital, por meio da aplicação de escalas, além da aferição da temperatura corporal pelo cateter na artéria pulmonar (padrão-ouro). Por fim, as Condutas de recuperação ou reabilitação evidenciaram o uso de medidas não-farmacológicas para o manejo da dor.

13. Arino M, Barrington JP, Morrison AL, Gillies D. Management of the changeover of inotrope infusions in children. Intensive Crit Care Nurs. 2004; 20 (5):275-80.

14. O’Brien P. A criança com disfunção cardiovascular. In: Hockenberry MJ, Wilson D, Inkelstein M. Wong Fundamentos de enfermagem pediátrica. 7th. ed. Rio de Janeiro: Elsevier; 2006. 15. Silva JP, Garanhani ML. O significado do cuidado perioperatório para a criança cirúrgica. Rev. Eletr. Enf. [Internet]. 2011 [cited 2012 dec 31];13(2):259-68. Available from:

http://dx.doi.org/10.5216/ree.v13i2.9917.

16. Harbaugh BL, Tomlinson PS, Kirschbaum M. Parents' perceptions of nurses' caregiving behaviors in the pediatric intensive care unit. Issues Compr Pediatr Nurs. 2004; 27(3):163-78.

17. Costa MCG, Arantes MQ, Brito MDC. A UTI Neonatal sob a ótica das mães. Rev. Eletr. Enf. [Internet]. 2010 [cited 2012 dec

31];12(4):698-704. Available from: http://dx.doi.org/10.5216/ree.v12i4.7130.

18. O'Brien P, Boisvert JT. Current management of infants and children with single ventricle anatomy. J Pediatr Nurs. 2001; 16(5): 338-50.

19. Puntillo KA, Jacob E. A survey of nursing practice in the assessment and management of pain in children. Pediatr Nurs.1999; 25(3):278-86.

20. Nethercott SG. The assessment and management of postoperative pain in children by registred sick children 's nurses: an exploratory study. J Clin Nurs. 1994; 3:109-14.

21. Joint Commission on Accreditation of Healthcare Organization, 2000 [cited 2010 June 17]. Available from: http://www.va.gov/oaa/pocketcard/pain5thvitalsign/PainToolkit Oc t2000.doc.

22. Oba J. Terapia nutricional na criança com cardiopatia congênita. In: Ebaid M. Cardiologia em pediatria: temas fundamentais. São Paulo: Rocca; 2000. p. 495-512.

23. João PRD, Faria Junior F. Cuidados imediatos no pós-operatório de cirurgia cardíaca. J Pediatr. 2003; 79(Supl.2):S213-S22.

Artigo recebido em 20/06/2011.

Aprovado para publicação em 02/04/2012.

Artigo publicado em 31/12/2012. 